\title{
TITLE:
}

\section{$<$ Note $>$ Notes on the Behavior of a Newly Immigrated Female Chimpanzee to the Mahale M group}

\section{AUTHOR(S):}

Nakamura, Michio; Itoh, Norio

\section{CITATION:}

Nakamura, Michio ...[et al]. <Note> Notes on the Behavior of a Newly Immigrated Female Chimpanzee to the Mahale M group. Pan Africa News 2005, 12(2): 20-22

\section{ISSUE DATE:}

2005-12

URL:

http://hdl.handle.net/2433/143455

\section{RIGHT:}

Copyright (C) Pan Africa News. 
Pan Africa News, 12(2), December, 2005

\section{$<$ NOTE $>$}

\section{Notes on the Behavior of a Newly Immigrated Female Chimpanzee to the Mahale M group.}

\author{
Michio Nakamura and Noriko Itoh
}

Kyoto University

\section{INTRODUCTION}

In chimpanzee societies, females usually transfer between unit-groups ${ }^{1}$ on reaching sexual maturity. Recent studies (e.g. 2) have revealed that cultural differences exist among chimpanzee groups. Although such studies usually compare groups that are geographically distant from each other, differences in the social customs between two neighboring groups have also been implied ${ }^{3}$.

Although direct comparisons among neighboring groups are often difficult, an observation of the behaviors of immigrant females might provide some insights. For example, some females showed idiosyncratic feeding habits ${ }^{4,5}$ or displayed subtly different types of grooming hand-clasp ${ }^{6}$, implying that these females retained the customs of their natal groups. If differences of customs do exist between two neighboring groups, then newly immigrated females should initially be unaware of the customs of the new group. Thus, an investigation of the behavior of newly immigrated females may be useful in determining how the behavioral repertoires differ between their natal groups and the new group. It may also provide information on which customs and the manner in which they are acquired by naïve individuals. However, because newly immigrated females are often intimidated by humans, there are few reports on the behaviors of such females immediately after their immigration.

Since we had an opportunity to observe the behavior of a newly immigrated female to the Mahale $\mathrm{M}$ group, we have stated our observations in this report.

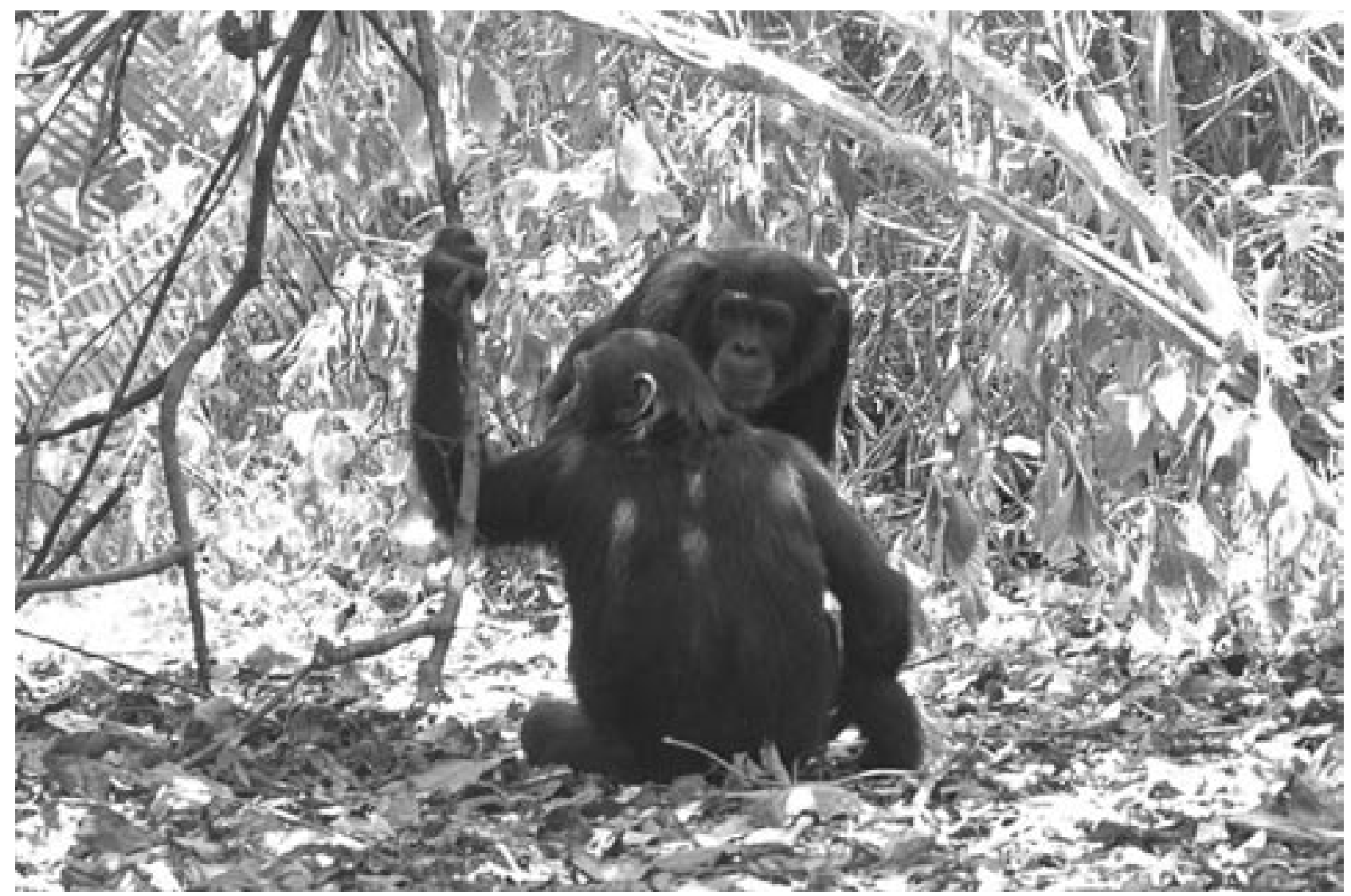

Figure 1. Yuri, on the second day after immigration, peering at Michio (an adolescent male) while on the observation trail. 


\section{OBSERVATIONS}

On September 24, 2005, when we found a party of the $M$ group chimpanzees at the middle of their home range, we noticed an unfamiliar young female on a tree. Since she had distinctive characteristics, we recognized her face thereafter and named her Yuri (Figure 1). Based on the size of her body and the sexual swelling, we estimated her age to be approximately ten to eleven years. We observed Yuri for approximately three hours intermittently on this day. The other $\mathrm{M}$ group members, particularly the males, appeared a little excited by the newcomer. However, we observed only one case of threatening behavior toward Yuri by an adult female, Fuji. Yuri copulated with at least four males, namely, Bonobo (an adult), Cadmus and Orion (adolescents), and Emory (an infant). She was groomed by Fuji (an adult female), Athena (an adolescent female), Acadia (a juvenile female), Masudi (an adult male), and Cadmus, Primus, and Christmas (adolescent males), however, she only groomed back Cadmus.

The grooming hand-clasp was reported as a social custom of both the extinct $\mathrm{K}$ group ${ }^{7}$ and the $M$ group $^{8}$ at Mahale. On the first day, Yuri performed the grooming hand-clasp with Cadmus on a tree. $\mathrm{S}$ he also performed leaf grooming ${ }^{9}$, which is another custom of the Mahale M group ${ }^{2}$.

On the next day, she even tolerated one of the observers up to a distance of approximately three meters, while she followed the other members on the observation trail. She even responded to the distant pant hoot by the members of the $M$ group. We also observed her playing with Michio (an adolescent male) on the ground.

During this season, Yuri's food repertoires did not differ greatly from that of the $M$ group chimpanzees, except, that she was not observed to eat lemons. Licking the surface of dead trees is another cultural behavior of the Mahale $M$ group $^{10}$. On November 12, 2005, we observed Yuri licking the dead wood of a Ficus vallis-choudae tree after several chimpanzees had licked it.

\section{DISCUSSION}

Yuri got accustomed to both humans as well as members of the $M$ group very quickly. This could be attributed to the possibility that she had immigrated well before we noticed her on that first day. However, she seemed quite unfamiliar with the $M$ group members as deduced from their unusual excitement on that day. Further, since only one habituated group inhabits Mahale at present, we cannot be certain of Yuri's natal group. Since she tolerated humans to some extent from the very beginning, there is a possibility that she had immigrated from the Miyako group to the north on which a habituation attempt is being conducted. However, she also ate Myrianthus arboreus fruits without hesitation although this fruit is scarce in the mountainous areas of the Miyako group. Another possibility is that she immigrated from the N'ganja group to the south, especially because most of the $M$ group members were in the area that overlaps with the N'ganja group's home range on the day before Yuri was first observed.

As she performed the grooming hand-clasp on the very first day without any hesitation, it is likely that she immigrated from a group where this behavior is customarily performed. Given the fact that chimpanzees from both $\mathrm{M}$ group and the extinct $\mathrm{K}$ group performed this behavior, the grooming hand-clasp could be a common culture of several neighboring groups in Mahale. It would be interesting to investigate further the extent to which this social custom is shared. Leaf grooming is also likely to be a custom of her natal group.

We could not observe Yuri eating lemons. Lemons were introduced to the $M$ group range by humans and have constantly been eaten by the $\mathrm{M}$ group chimpanzees since $1982^{11}$. Thus, it is likely that chimpanzees of other groups in Mahale might not be aware of the fruit. Due to a scarcity of lemon fruits during the period after she immigrated to the $M$ group, we did not have an opportunity to observe how Yuri dealt with the unfamiliar fruits when the other members were feeding on them. As most immigrant females do eat lemons, it may also be interesting to follow the process of how such females learn to eat the unfamiliar fruit. 


\section{ACKNOWLEDGIMENTS}

The field research was supported by grants from the Japanese MEXT (\#16255007 to T. Nishida, \#14083205 to K. Sugawara, \#16770186 to M. Nakamura) and Biodiversity Research of the 21st Century COE (A14).

\section{REFERENCES}

1. Nishida T and Kawanaka K. 1972. Inter-unitgroup relationships among wild chimpanzees of the Mahali Mountains. Kyoto University African Studies 7:131-169.

2. Whiten A, Goodall J, McGrew WC, Nishida T, Reynolds V, Sugiyama Y, Tutin CEG, Wrangham RW and Boesch C. 1999. Cultures in chimpanzees. Nature 399:682-685.

3. McGrew WC, Marchant LF, Scott SE and Tutin CEG. 2001. Intergroup differences in a social custom of wild chimpanzees: the grooming hand-clasp of the Mahale Mountains. Current Anthropology 42:148-153.

4. Matsuzawa T. 1994. Field experiments of use of stone tools in the wild. In: Chimpanzee Cultures. Wrangham WR et al. (eds), Harvard Univ Press, Cambridge, Mass., pp. 351-370.

5. Sakamaki T. 1998. First record of algae-feeding by a female chimpanzee at Mahale. Pan Africa News 5:1-3.

6. Nakamura M and Uehara S. 2004. Proximate factors of two types of grooming-hand-clasp in Mahale chimpanzees: implication for chimpanzee social custom. Current Anthropology 45:108-114.

7. McGrew WC and Tutin CEG. 1978. Evidence for a social custom in wild chimpanzees? Man 13:234-251.

8. Nakamura M. 2002. Grooming-hand-clasp in Mahale $M$ group chimpanzees: implication for culture in social behaviors. In: Behavioural Diversity in Chimpanzees and Bonobos. Boesch C et al. (eds), Cambridge University Press, Cambridge, pp. 71-83.

9. Zamma K. 2002. Leaf-grooming by a wild chimpanzee in Mahale. Primates 43:87-90.

10. Nishida T, Kano T, Goodall J, McGrew WC and Nakamura M. 1999. Ethogram and ethnography of Mahale chimpanzees. Anthropological Science 107:141-188.

11. Takahata Y, Hiraiwa-Hasegawa M, Takasaki H and Nyundo R. 1986. Newly acquired feeding habits among the chimpanzees of the Mahale Mountains National Park, Tanzania. Human Evolution 1:277-284. 